\title{
Estudos farmacognósticos, fitoquímicos, atividade antiplasmódica e toxicidade em Artemia salina de extrato etanólico de folhas de Montrichardia linifera (Arruda) Schott, Araceae
}

\author{
Eliene S. S. Costa, ${ }^{1}$ Maria Fâni Dolabela, ${ }^{* 2,3}$ Marinete M. Póvoa, ${ }^{3}$ Davi J. Oliveira, ${ }^{2}$ \\ Adolfo H. Müller ${ }^{1,2}$
}

\author{
${ }^{1}$ Departamento de Química, Centro de Ciências Exatas e Naturais, Universidade Federal do Pará, \\ Rua Augusto Corrêa, 1, 66075-110 Belém-PA, Brasil \\ ${ }^{2}$ Curso de Farmácia, Área de Ciências Biológicas e da Saúde, Centro Universitário do Pará, Av. Nazaré, 630, \\ 66035-170 Belém-PA, Brasil \\ ${ }^{3}$ Seção de Parasitologia, Instituto Evandro Chagas s/n, 67030-000 Ananindeua-PA, Brasil
}

\begin{abstract}
RESUMO: O presente trabalho descreve os resultados do estudo farmacognóstico, estudo fitoquímico preliminar, toxicidade em Artemia salina e atividade antiplasmódica contra cepa de Plasmodium falciparum resistente a cloroquina (W2) do extrato etanólico obtido do pó das folhas de Montrichardia linifera (Arruda) Schott, Araceae. Para realização dos estudos farmacognósticos utilizou-se as metodologias descritas na Farmacopéia Brasileira. A prospecção fitoquímica foi realizada pelos métodos descritos por Mattos. No ensaio antiplasmódico foi utilizado o microteste tradicional e no ensaio de Artemia salina. Os resultados obtidos nos estudos farmacognósticos do pó das folhas de $M$. linifera demonstraram tratar-se de um pó moderadamente grosso, com teores de água e cinzas totais dentro dos limites aceitáveis, baixa densidade $(0,324$ e 0,339 g/ $\mathrm{mL}), \mathrm{pH}$ próximo de neutro $(6,69 \pm 0,02)$. A análise fitoquímica preliminar evidenciou a presença de alcaloides, flavonoides, taninos, triterpenos e esteroides. O extrato etanólico das folhas de $M$. linifera apresentou moderada atividade antiplasmódica e baixa toxicidade para a Artemia salina.
\end{abstract}

Unitermos: Montrichardia linifera, estudos farmacognósticos, atividade antiplasmódica, toxicidade, Artemia salina.

\begin{abstract}
Pharmacognostics studies, phytochemicals, antiplasmodic activity and toxicity in Artemia salina of ethanolic extract from Montrichardia linifera (Arruda) Schott, Araceae leaves". The present paper describes results of a pharmacognostic, a preliminary phytochemical study, brine shrimp toxicity and antiplasmodic activity in a strain of Plasmodium falciparum that is resistant to chloroquine (W2) using ethanolic extract obtained from powder of the leaves of Montrichardia linifera (Arruda) Schott, Araceae. To perform the pharmacognostic studies methodologies employed in the Brazilian Pharmacopoea were utilized. The methods described by Mattos were utilized for phytochemical prospecting. In the antiplasmodic test the traditional microtest was employed, as well as in the test with brine shrimp. The results obtained in the pharmacognostic studies with the powder from $M$. linifera leaves showed that it was a moderate thick powder, with water and ash totals within acceptable limits, low density $(0.324$ and $0.339 \mathrm{~g} /$ $\mathrm{mL}), \mathrm{pH}$ close to neutral $(6.69 \pm 0.02)$. Preliminary phytochemical analysis showed the presence of alkaloids, flavonoids, tannins, triterpenes and esters. The ethanolic extract from $M$. linifera leaves presented moderate antiplasmodic activity and low brine shrimp toxicity. To summarize, the powder used in preparing the extract presented good quality in pharmacognostic terms.
\end{abstract}

Keywords: Montrichardia linifera, pharmacognostic studies, antiplasmodic activity and brine shrimp toxicity, Artemia salina.

\section{INTRODUÇÃO}

Montrichardia linifera (Arruda) Schott, pertence à família Araceae, que é composta por 110 gêneros e aproximadamente 2500 espécies, de distribuição predominantemente nos trópicos. Na amazônia brasileira, os elementos ribeirinhos mais representativos são as espécies do gênero Montrichardia Crueg: M. linifera (Arruda) Schott e M. arborescens (L.) Schott. M. linifera é conhecida popularmente como "aninga", "aningaçu", "aningaíba" ou ainda "aninga-do-igapó" (Lins, 1994). M. linifera distribuí-se pelas margens e 
desembocaduras dos rios e igarapés, formando densas populações, oferecendo resistência ao movimento das águas (Hubber, 1943). É uma planta pioneira, por colonizar os solos nus das margens dos rios, fazendo com que esta espécie desempenhe um papel importante na preservação dessas margens (Macedo et al., 2005).

Para M. linifera foi descrita sua utilização como expectorante (Lins, 1994), suas folhas são consideradas anti-reumáticas e efetivas em úlceras; suas raízes são antidiuréticas apesar de tóxicas e suas cinzas são ricas em potássio (Plowman, 1969).

A composição química de $M$. linifera ainda não é conhecida e também não foram realizados estudos biológicos e farmacológicos. O presente trabalho descreve as características farmacognósticas das folhas de $M$. linifera, resultados de estudos fitoquímicos preliminares realizados com o extrato etanólico obtido do pó das folhas, atividade antiplasmódica e toxicidade em Artemia salina deste extrato.

\section{MATERIAL E MÉTODOS}

\section{Material vegetal}

As folhas Montrichardia linifera (Arruda) Schott Araceae foram coletadas no Campus da Universidade Federal do Pará, em Belém-PA, às margens do rio Guamá, $01^{\circ} 28^{\prime} 41,3^{\prime \prime} \mathrm{S}$ e $48^{\circ} 27^{\prime} 29,0^{\prime \prime} \mathrm{W}$, em abril de 2007 . A identificação do material foi realizada pela Dr. Alba Lúcia Ferreira de Almeida Lins do Departamento de Botânica do Museu Paraense Emilio Goeldi (Belém-PA). Uma exsicata da espécie foi depositada no herbário João Murça Pires, MPEG, sob o número 186542.

\section{Preparo, secagem do material vegetal e obtenção do extrato etanólico}

O material vegetal (lâmina foliar) foi seco em estufa com desumidificador à aproximadamente $45{ }^{\circ} \mathrm{C}$ por sete dias e moído em moinho de facas. Após secagem e moagem foi obtido o pó das folhas de M. linifera. Parte deste pó $(400 \mathrm{~g})$ foi reservado para os estudos farmacognósticos e o restante $(410 \mathrm{~g})$ foi submetido à percolação descontínua com etanol, seguida de filtração e concentração em evaporador rotativo, para obtenção do extrato etanólico.

\section{Análise granulométrica}

Cerca de $25 \mathrm{~g}$ de pó das folhas secas de $M$. linifera foram colocados sobre um conjunto de tamises de malhas de abertura nominal $1,70 \mathrm{~mm}, 710 \mu \mathrm{m}, 250 \mu \mathrm{m}, 180 \mu \mathrm{m}$, $125 \mu \mathrm{m}$, provido de tampa e fundo coletor. O conjunto de tamises foi colocado em tamisador vibratório e submetido à passagem forçada por vibração na escala sete do aparelho, durante $30 \mathrm{~min}$ em triplicata, primeiramente e depois por $1 \mathrm{~h}$ e $30 \mathrm{~min}$. Após estes procedimentos realizou-se a pesagem das frações retiradas dos tamises e do fundo coletor (F. Bras., 1988).

\section{Determinação da densidade bruta do pó das folhas}

Para determinar a densidade bruta do pó foram utilizados os seguintes métodos:

\section{Método da proveta}

O pó da droga foi transferido para uma proveta de $25 \mathrm{~mL}$, com peso conhecido, até completar o volume de $15 \mathrm{~mL}$. Durante a transferência foram removidas as partículas de ar que estavam presentes entre o pó da planta. Em seguida, a proveta contendo o pó foi pesada, e pela diferença de pesos, foi obtida a quantidade de pó contido na proveta. Com essas informações, o peso do pó dividido pelo volume $(15 \mathrm{~mL})$, foi calculado a densidade. Este procedimento foi realizado em triplicata (Lachman, 2001).

\section{Método do peso médio das cápsulas}

O pó da planta ( $15 \mathrm{~g})$ foi utilizado para preencher quarenta cápsulas de tamanho $00(0,95 \mathrm{~mL})$. Vinte cápsulas, escolhidas aleatoriamente, foram pesadas cheias e vazias. Pela diferença dos pesos foi calculada a quantidade do pó que cada cápsula continha. Em seguida, o peso médio do pó contido nas cápsulas dividido pelo volume $(0,95$ $\mathrm{mL}$ ) permitiu calcular a densidade. Este experimento foi repetido três vezes com três amostras de pó distintas (Martins \& Sacramento, 2004).

\section{Perda por secagem da folha fresca}

Foram pesados exatamente $2 \mathrm{~g}$ de material vegetal que foram transferidos para pesa-filtro chato, dessecado, nas mesmas condições do teste a ser realizado, até peso constante. O pesa-filtro contendo a amostra foi pesado e tampado. Em seguida, o mesmo foi agitado para uniformizar a amostra. Colocou-se o pesa-filtro destampado juntamente com a tampa na estufa. Secou-se a amostra a $105-110{ }^{\circ} \mathrm{C}$, primeiramente por $5 \mathrm{~h}$, e depois, de hora em hora, até peso constante, ou seja, diferença entre pesagens menores do que $0,5 \mathrm{mg}$ (F. Bras., 2000). O resultado foi expresso pela média de quatro determinações.

\section{Determinação do teor de cinzas}

Foram pesados exatamente $3 \mathrm{~g}$ de material vegetal e transferidos para cadinho de porcelana, previamente calcinado a $450{ }^{\circ} \mathrm{C} \mathrm{em}$ forno mufla, estabilizado sob as mesmas condições do teste. O material foi uniformizado no cadinho e submetido à calcinação por uma hora, resfriado em dessecador por 30 min e pesado em balança

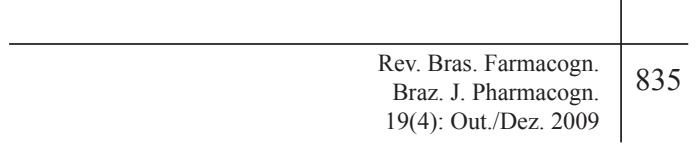


analítica. Este procedimento foi repetido até a diferença de peso entre duas pesagens consecutivas não ultrapassar 0,5 mg (F. Bras., 2000).

\section{Determinação de pH}

A determinação de $\mathrm{pH}$ foi realizada de acordo com a F. Bras. (1988), preparando uma solução $1 \%(\mathrm{p} / \mathrm{v})$ do pó da folha em água destilada, aquecida até ebulição em chapa-elétrica por 5 min. A solução resultante foi filtrada utilizando algodão. Após o resfriamento foi medido o $\mathrm{pH}$ do filtrado utilizando um potenciômetro previamente calibrado. Os resultados foram obtidos pela média de três determinações.

\section{Determinação de substâncias extraíveis em etanol}

Exatamente $2 \mathrm{~g}$ de pó vegetal foram transferidos para o cartucho de um extrator de Soxhlet, previamente tarado e seco. No balão do extrator foram colocados 200 $\mathrm{mg}$ de hidróxido de sódio e $500 \mathrm{~mL}$ de etanol absoluto. Após cinco horas, quando o processo de extração foi encerrado, o cartucho com o resíduo foi retirado e seco em estufa a $105^{\circ} \mathrm{C}$, por $30 \mathrm{~min}$. Pesou-se o resíduo seco e calculou-se o teor de substâncias extraíveis por etanol por diferença entre o peso da amostra e o peso do resíduo seco (F. Bras., 2000)

\section{Análise fitoquímica preliminar}

Essas análises visaram o conhecimento de possíveis grupos químicos presentes no extrato etanólico. Através de reações químicas pesquisou-se a presença de alcaloides, antraquinonas, cumarinas, flavonoides, saponinas, triterpenos e esteroides (Mattos, 2000).

\section{Atividade antiplasmódica}

Cultivo do Plasmodium falciparum

O cultivo foi realizado de acordo com Trager \& Jensen (1976). A cultura do clone de P. falciparum W2 foi mantida em estufa a $37^{\circ} \mathrm{C}$, em atmosfera de 3 a $5 \%$ de $\mathrm{CO}_{2}$, conseguida através da queima de vela, em dessecador. A parasitemia foi diariamente determinada e, quando maior que $6 \%$, a placa era submetida à sincronização com sorbitol e utilizada para a realização do microteste.

\section{Microteste tradicional}

A metodologia utilizada neste ensaio foi descrita por Rieckman et al. (1978) e modificada por Carvalho (1990). O extrato foi solubilizado com uma solução contendo meio RPMI e $0,025 \%$ (v/v) de dimetilssulfóxido, sendo utilizadas as seguintes concentrações: $3,125,6,25$, $12,5,25,50,100 \mu \mathrm{g} / \mathrm{mL}$. Como controle positivo foi utilizado cloroquina em diferentes concentrações.

Cultivos sincronizados contendo, predominantemente, trofozoítos $(>90 \%)$, foram diluídos para $0,5 \%$, o hematócrito ajustado para $2,5 \%$ e distribuído em placas contendo o extrato ou a cloroquina. Após 24 e $48 \mathrm{~h}$ trocava-se o meio adicionando-se nova dose do extrato ou da cloroquina. Após $72 \mathrm{~h}$ foram confeccionados os esfregaços, determinando-se a parasitemia percentual. De posse das parasitemias percentuais, calculou-se a $\mathrm{CI}_{50}$ utilizando-se o método Graph Pad Prism 4,0. Os resultados foram expressos baseados na média de dois experimentos.

\section{Toxicidade em Artemia salina}

Cerca de dez larvas de Artemia salina foram transferidas para tubos de ensaios contendo água artificial do mar e o extrato etanólico, em cinco diferentes concentrações cada uma em triplicata constituindo um ensaio, este ensaio foi realizado três vezes. A contagem dos animais mortos e vivos foi realizada após $24 \mathrm{~h}$. Utilizouse o método Probitos de análise para obtenção das $\mathrm{DL}_{50} \mathrm{e}$ respectivos intervalos de confiança (Meyer et al., 1982).

\section{RESULTADOS}

No presente trabalho, a padronização farmacognóstica do pó das folhas de Montrichardia linifera (Arruda) Schott Araceae foi iniciada pela avaliação granulométrica, que é um parâmetro a ser estabelecido devido influenciar diretamente no processo, sendo classificado como moderadamente grosso. Os resultados das análises encontram-se na Tabela 1.

A densidade foi de $0,324 \pm 0,02$ pelo método da proveta e de $0,339 \pm 0,009$ pelo método do peso médio das cápsulas, não sendo observada diferença significativa $(\mathrm{p}=$ 0,3003 , teste t-Student) entre os valores obtidos pelos dois métodos (Martins \& Sacramento, 2004). O teor de água foi de $7,4 \% \pm 0,07$, estando dentro dos limites aceitáveis (F. Bras., 2000), pois alto teor de água residual no pó da planta pode propiciar a contaminação por microorganismos e degradação dos componentes químicos. $\mathrm{O}$ pó da planta apresentou teor de cinzas totais de $8,1 \% \pm 0,08$. Este percentual constitui a matéria inorgânica não volátil que pode estar como contaminante do pó da planta. O valor encontrado está dentro dos limites aceitáveis pela F. Bras. (1988) (Tabela 2).

$\mathrm{O} \mathrm{pH}$ foi de $6,69 \pm 0,02$, sugerindo que o pó desta planta deva conter predominantemente substâncias neutras (Migliato et al., 2007). O teor de extrativos em etanol (F. Bras., 2000) foi de $9,02 \% \pm 0,88$ (Tabela 2).

A abordagem fitoquímica do extrato etanólico obtido das folhas de $M$. linifera revelou a presença de alcaloides, flavonoides, taninos, esteroides e triterpenoides. O extrato apresentou baixa toxicidade para a Artemia salina $\left(\mathrm{DL}_{50}>500 \mu \mathrm{g} / \mathrm{mL}\right)$ e para a atividade antiplasmódica apresentou moderada atividade $\left(10<\mathrm{CI}_{50}<100 \mu \mathrm{g} / \mathrm{mL}\right)$ (Tabela 3). 
Tabela 1. Análise da granulometria do pó das folhas de $M$. linifera.

\begin{tabular}{ccccc}
\hline \multirow{2}{*}{$\begin{array}{c}\text { Abertura nominal } \\
\text { da malha }\end{array}$} & \multicolumn{4}{c}{ Amostra (\%) } \\
\cline { 2 - 5 } & 1 & 2 & 3 & 4 \\
\hline $1,70 \mathrm{~mm}$ & 43,32 & 24,16 & 28,08 & 43,32 \\
$850 \mu \mathrm{m}$ & 3,20 & 3,40 & 3,44 & 3,08 \\
$250 \mu \mathrm{m}$ & 39,08 & 51,12 & 49,84 & 37,76 \\
$180 \mu \mathrm{m}$ & 4,76 & 6,04 & 8,28 & 6,52 \\
$125 \mu \mathrm{m}$ & 3,36 & 4,24 & 3,64 & 1,72 \\
Fundo coletor & 5,84 & 10,52 & 6,48 & 6,60 \\
\hline
\end{tabular}

Tabela 2. Análises farmacognósticas do pó das folhas de $M$. linifera.

\begin{tabular}{lc}
\hline Parâmetro & Resultados obtidos \pm desvio padrão \\
\hline Densidade do pó & \\
a)-Método da proveta & $0,324 \pm 0,02 \mathrm{~g} / \mathrm{mL}$ \\
b)-Peso das cápsulas & $0,339 \pm 0,009 \mathrm{~g} / \mathrm{mL}$ \\
Perda por dessecação & $7,4 \% \pm 0,07$ \\
Teor de cinzas totais & $8,1 \% \pm 0,08$ \\
pH & $6,69 \pm 0,02$ \\
Teor de extrativos em & $9,02 \% \pm 0,88$ \\
etanol & \\
Índice de espuma & $0,36 \pm 0,11 \mathrm{~cm}$ \\
\hline
\end{tabular}

Tabela 3. Análise fitoquímica, atividade antiplasmódica e toxicidade em $A$. salina do extrato etanólico de folhas de $M$. linifera.

\begin{tabular}{cc}
\hline \multicolumn{2}{c}{ Análises fitoquímicas preliminares } \\
\hline $\begin{array}{c}\text { Metabólitos presentes na } \\
\text { planta }\end{array}$ & $\begin{array}{c}\text { Alcaloides, flavonoides, } \\
\text { taninos, triterpenos e } \\
\text { esteroides. }\end{array}$ \\
\hline Atividade antiplasmódica \\
\hline $\mathrm{W}_{2}-\mathrm{CI}_{50}$ \\
\hline Toxicidade em Artemia salina \\
\hline $\mathrm{DL}_{50}$
\end{tabular}

Legenda: W2- clone de $P$. falciparum resistente a cloroquina e sensível a mefloquina; $\mathrm{CI}_{50}$ - concentração inibitória $50 \%$; $\mathrm{DL}_{50}{ }^{-}$ dose letal $50 \%$; *-desvio padrão.

\section{DISCUSSÃO}

Para assegurar a qualidade de um medicamento fitoterápico, é essencial o controle da matéria-prima feita através de ensaios, os quais irão estabelecer parâmetros para o material em estudo através da determinação do teor de umidade, cinzas totais, granulometria, $\mathrm{pH}$, densidade e índice de espuma, os quais garantirão um padrão para a planta a ser utilizada (Newall et al., 2002). No presente estudo, as condições adotadas na coleta, secagem e moagem contribuíram para a qualidade da matéria-prima, visto que em todos os estudos farmacognósticos foram obtidos resultados dentro do limite aceitável pela F. Bras. (2000).
Além disso, também é necessário conhecer a composição química do extrato que utiliza testes químicos qualitativos rápidos e de baixo custo (Mattos, 2000) e sugere as possíveis classes de metabólitos secundários de interesse farmacológico que estão presentes nos extratos, para que se possa delinear o melhor método para sua extração e os bioensaios que devem ser submetidos. A presença de alcaloides e triterpenos no extrato etanólico das folhas de Montrichardia linifera (Arruda) Schott Araceae, indicada pela abordagem fitoquímica, sugeriu para o referido extrato uma potencial atividade antiplasmódica (Basco et al., 1994). Então foi realizado o ensaio para atividade antiplasmódica utilizando um clone resistente a cloroquina e observou-se moderada atividade (Tabela 3).

$\mathrm{O}$ teste de toxicidade sobre a Artemia salina (TAS) é um ensaio biológico amplamente utilizado devido ser rápido, confiável, de baixo custo e por ter demonstrado uma boa correlação com várias atividades biológicas (Meyer et al., 1982), como atividade antitumoral (McLaughlin, 1991; McLaughlin et al., 1991, 1993), atividade contra o Trypanosoma cruzi (Dolabela, 1997), atividade antibacteriana (Brasileiro et al., 2006; Niño et al., 2006; Magalhães et al., 2007), antifúngico (Niño et al., 2006; Magalhães et al., 2007). Em geral, extratos com alta toxicidade para Artemia salina $\left(\mathrm{DL}_{50}<200 \mu \mathrm{g} / \mathrm{mL}\right)$ apresentam alto potencial para estas atividades. $\mathrm{O}$ extrato de M. linifera apresentou baixa toxicidade para Artemia salina $\left(\mathrm{DL}_{50}>5000 \mu \mathrm{g} / \mathrm{mL}\right.$ ) (Tabela 3), sugerindo que talvez não tenha potencial antitumoral, tripanossomicida, antibacteriano e antifúngico.

\section{AGRADECIMENTOS}

Ao Centro Universitário do Estado do Pará (CESUPA) pela disponibilidade da sua infraestrutura laboratorial que permitiram a realização integral deste trabalho.

\section{REFERÊNCIAS}

Basco LK, Mitaku S, Skaltsounis AL, Ravelomanantsoa N, Tillequin F, Koch M, Le Bras J 1994. In vitro activities of furoquinoline and acridone alkaloids against Plasmodium falciparum. Antimicrob Agents Ch 38: 1169-1171.

Brasileiro BG, Pizziolo VR, Raslan DS, Jamal CM, Silveira D 2006. Antimicrobial and cytotoxic activities screening of some Brazilian medicinal plants used in Governador Valadares district. Rev Bras Cienc Farm 42: 195-202.

Carvalho LH 1990. Quimioterapia experimental com extratos brutos de plantas e compostos quimicamente definidos. Minas Gerais, 146p. Dissertação de mestrado, Departamento de Parasitologia, ICB, Universidade Federal de Minas Gerais.

Dolabela MF 1997. Triagem in vitro para atividade antitumoral e anti Trypanossoma cruzi de extratos vegetais, produtos naturais e susbstâncias sintéticas. Minas Gerais, 130p. 
Dissertação de Mestrado, Departamento de Fisiologia e Farmacologia, ICB, Universidade Federal de Minas Gerais.

Farmacopéia Brasileira 1988. 4. ed. São Paulo, Atheneu.

Farmacopéia Brasileira 2000. 4. ed. São Paulo, Atheneu.

Hubber J 1943. Contribuição e geografia física da parte Ocidental do Marajó. Rev Bras Geogr 5: 449-474.

Lachman L, Lieberman HA, Kanig JL 2001. Teoria e Prática na Indústria Farmacêutica. Lisboa: Calouste Gulbenkian.

Lins ALFA 1994. Aspectos morfológicos e anatômicos de raízes do gênero Montrichardia Crüger. (Araceae). 91p. Dissertação de Mestrado em Ciências Biológicas/ Botânicas, Universidade Federal do Rio Grande do Sul.

Macedo EG, Filho BGS, Potiguara RCV, Santos DSB 2005. Anatomia e arquitetura foliar de Montrichardia linifera (Arruda) Schott (Araceae). Espécie da Várzea Amazônica. Bol Mus Para Emílio Goeldi Cienc Nat 1: 19-43.

Magalhães AF, Tozzi AMGA, Santos CC, Serrano DR, ZanottiMagalhães EM, Magalhães EG, Magalhães LA 2007. Saponins from Swartzia langsdorffii: biological activities. Mem Inst Oswaldo Cruz 98: 713-718.

Martins HB, Sacramento LVS 2004. Análise microscópica e física para controle de qualidade de matéria prima vegetal pulverizada. $16^{\circ}$ Congresso de Iniciação Científica, UNESP, Ilha Solteira, Brasil.

Mattos FJA 2000. Plantas medicinais: guia de seleção e emprego de plantas usadas em fitoterapia no Nordeste do Brasil. 2. ed. Fortaleza: IU.

McLaughlin JL 1991. Crown gall tumours on potato discs and brine shrimp lethality: two simple bioassays for higher plant screening and fractions. In: Dey PM, Harbone JB (ed.) Methods in Plant Biochemistry. New York: Academic Press, p. 1-32.

McLaughlin JL, Chang CJ, Smith DL 1991. "Bench-top" bioassays for the discovery of bioactive natural products: an update. In: Rahman A(ed.) Studies in Natural Product Chemistry 9. Amsterdam: Elsevier, p. 383-409.

McLaughlin JL, Chang CJ, Smith DL 1993. Simple bench-top bioassays (BS \& PD) for discovery of plant antitumor compounds-review of recent progress in human medicinal agents from plants. Nova York: Kinghorn \& Balandrini, p. 112-137.

Meyer BN, Ferrigini NR, Putnan JE, Jacobsen LB, Nichols DE, Mclaughlin JL 1982. Brine shrimp: a convenient general bioassay for active plants constituents. Planta Med 45: 31.

Migliato KF, Moreira RRD, Mello JCP, Sacramento LVS, Correa MA, Salgado HRN 2007. Controle de qualidade do fruto Syzyguim cumini (L.) Skeels. Rev Bras Farmacogn 17: 94-102

Newall CA, Anderson LA, Phillipson JD 2002. Plantas medicinais-Guia para profissional de saúde. São Paulo: Premier.

Niño J, Narváez DM, Mosquera OM, Correa YM 2006. Antibacterial, antifungal and cytotoxic activities of eight Asteraceae and two Rubiaceae plants from colombian biodiversity. Braz J Microbiol 37: 566-570.

Plowman T 1969. Folk uses of nem world aroids. Econ Bot 23: 97-122.

Rieckmann KH, Sax LJ, Campbell GH, Mrena JF 1978. Drug sensitity of Plasmodium falciparum. An in vitro microtechnique. Lancet 1: 22-23.

Trager W, Jensen JB 1976. Human malaria parasites in contiuous culture. Science 193: 673-675. 